\title{
Acute stroke after scalp injection of platelet rich plasma and stem cells for hair loss
}

\section{Abstract}

We present the case of a 57 year-old-right handed man with a history of alopecia presented with a visual loss, and an acute stroke of the left anterior cerebral artery and middle cerebral after scalp injection of platelet rich plasma (PRP) and stem cells for hair restoration.

Keywords: Alopecia, Visual loss, Acute stroke, Anterior cerebral artery, middle cerebral artery, Platelet rich plasma, Stem cells, Hair restoration, Embolic strokes
Volume 3 Issue 6 - 2015

Aldo F Berti, ' Alejandro Santillan, ${ }^{2}$ Pamela Garcia-Corrochano ${ }^{3}$

'Miami Neurosurgical Center, South Miami, USA

${ }^{2}$ Department of Neurology, University of Texas Health Science Center at San Antonio, USA

${ }^{3}$ Instituto de Neurocirugía, Neurología y Rehabilitación, Peru

Correspondence: Alejandro Santillan, MD, Department of Neurology, University of Texas Health Science Center at San Antonio, 8300 Floyd Curl Drive MSC 7883, San Antonio, TX 78229, USA, Tel 703-798-2246,

Email alejandrosantillan@gmail.com

\section{Case report}

A 57 year-old-right handed man with a history of alopecia areata presented from an outside facility after scalp injection of platelet rich plasma (PRP) and stem cells for hair restoration. The injection was administered behind the hairline in the frontal scalp. After the procedure, he started having severe headaches followed by transient loss of consciousness, complete loss of vision in the left eye and right-sided hemiparesis as well as mild dysarthria. NIHSS score at presentation was 8 . The patient was taken to the ICU where he stayed under observation for 5 days. An MRI of the brain after the procedure showed acute stroke involving the left anterior cerebral artery (ACA) and middle cerebral artery (MCA) territories (Figure 1). The patient also developed necrosis of the scalp in the territory of the left supratrochlear artery (Figure 2). He is slowly recovering from the stroke and currently receiving rehabilitation.
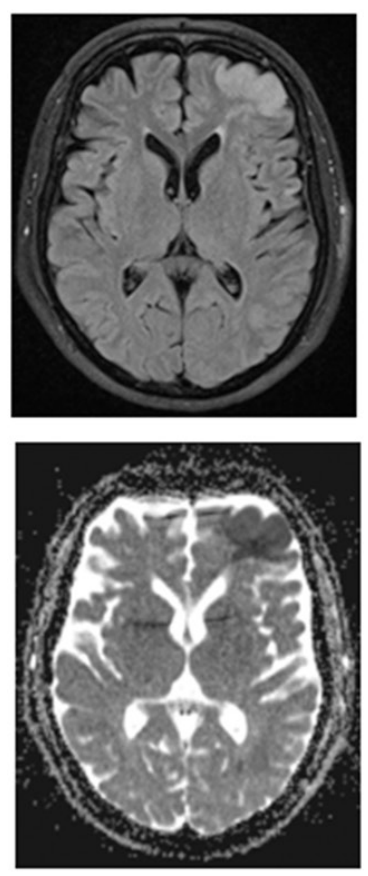

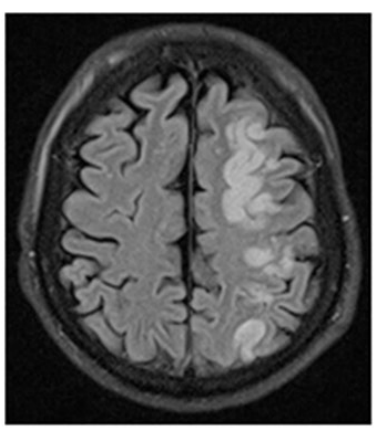

C

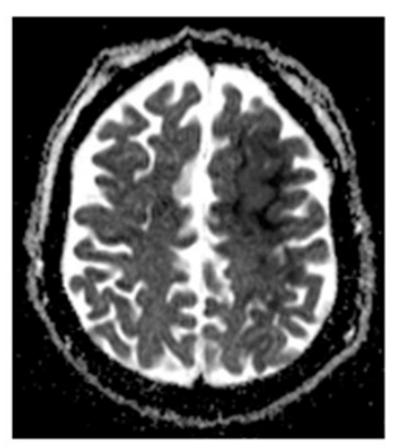

D

Figure I FLAIR MRI images and corresponding ADC maps show acute stroke in the ACA (Figure IA and B) and MCA territories (Figure IC and D).

\section{Comment}

Platelet rich plasma (PRP) and stem cell therapy are currently used as a promising new therapy for hair loss in centers for hair restoration worldwide. In the case presented, stem cells were extracted from the abdominal fat of the patient. Immediately after the procedure, the patient developed acute vision loss and embolic infarction of the ACA and MCA territories probably related to reverse flow of the injected material into the left supratrochlear artery causing occlusion of the left ophthalmic artery through reverse flow, ${ }^{1}$ to finally migrate into the left ACA and MCA territories via the internal carotid artery. One month later the patient developed necrosis of the scalp at the level of the left 
supratrochlear artery. The technique for the extraction of stem cells from the abdominal fat in this report is unknown and we believe that the injected material included adipose tissue that might have caused embolic strokes.

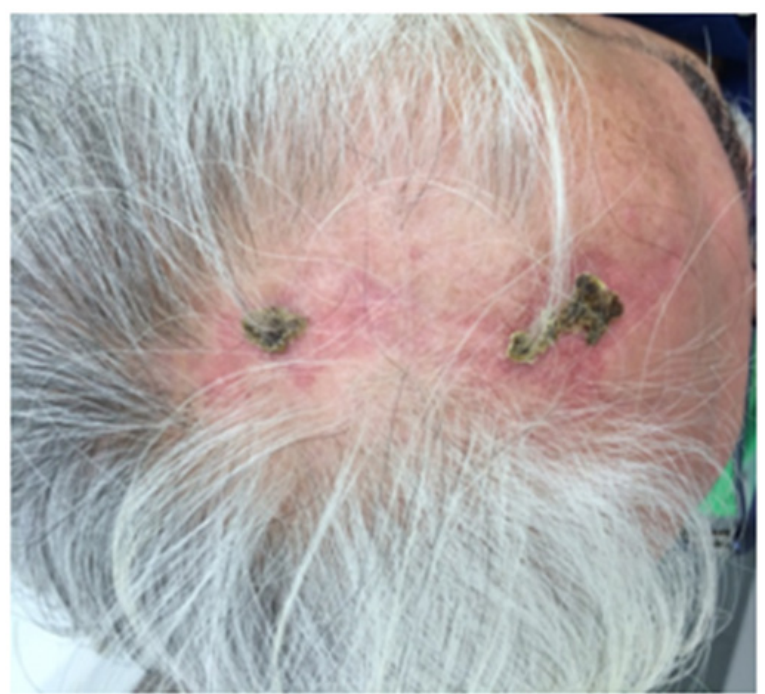

Figure 2 Necrosis of the scalp at the level of the left supratrochlear artery I month after injection of PRP and stem cells for hair restoration

\section{Acknowledgments}

None.

\section{Conflicts of interest}

None.

\section{References}

1. Hayreh SS. Orbitalvascularanatomy. Eye (Lond). 2006;20(10):11301144. 